\title{
A p-Laplacian Random Walk: Application to Video Games
}

\author{
Nicolas Wicker \\ Dépt. de Mathématiques \\ University of Lille
}

\author{
Canh Hao Nguyen \\ Bioinformatics Center \\ Inst. for Chemical Research \\ Kyoto University
}

\author{
Hiroshi Mamitsuka \\ Bioinformatics Center \\ Inst. for Chemical Research \\ Kyoto University
}

\begin{abstract}
A graph random walk is presented. It is derived from the $p$-Laplacian similarly to the derivation of the canonical random walk from the Laplacian. This variant enables quicker exploration while still sticking to the connection constraints given by the graph. A potential application is shown for video games.
\end{abstract}

Keywords: graph Laplacian, p-Laplacian, random walk, video games.

\section{Random walks on a graph and Laplacian}

Random walk on graphs are a classical subject in probability (Grimmet and Stirzaker 2004), statistics (von Luxburg, Radl, and Hein 2014) and algebra (Żuk and Pak 2002). The standard random walk is defined as a Markov chain where the first order transition probability from one vertex to another is given by $1 / d$ where $d$ is the degree of the first vertex. The stationary distribution of this Markov chain is given by $\pi=\frac{1}{m}\left(d_{1}, \ldots, d_{n}\right)$ where $m=\sum_{i=1}^{n} d_{i}$. In some cases, as video games it may be desirable to have a random walk starting at some vertex. Some variants of it would be to explore more quickly the graph. Let's say there is a monster exploring rooms in a dungeon where rooms are connected to each other. At each step, a monster can only move to a neighbour room. So, to accelerate its visits, one way would be to count more iterations but this would have the drawback of making the monster jump between rooms. Consequently, here we propose an alternative way consisting in going back to the definition of the canonical random walk through its associated graph Laplacian and achieving that way our goal.

The idea is to modify slightly the random walk going back to its definition by a Laplacian. The Laplacian can be considered in two equivalent ways, either as something proportional to the infinitesimal generator or as an operator to be minimized when going from one time step to another. Let us recall first the definition of the infinitesimal generator of the Markov chain $X$ where $X_{x}^{t}$ is the state at time $t$ of the Markov chain starting at $x$ at time 0 . The infinitesimal generator in continuous time for a multivariate diffusion in $\mathbb{R}^{p}$ as the operator 
on a function $f: \mathbb{R}^{p} \rightarrow \mathbb{R}$ is then given by:

$$
A f(x)=\lim _{t \rightarrow 0} \frac{\mathbf{E}\left[f\left(X_{x}^{t}\right)\right]-f(x)}{t}
$$

In can be discretized in many ways, one of them is the following:

$$
\begin{aligned}
A f(i) & =\sum_{j \mid j \sim i} w_{i j} \frac{f(j)}{d_{i}}-f(i) \\
& =\frac{1}{d_{i}} \sum_{j \mid j \sim i} w_{i j}[f(j)-f(i)] \\
& =-\frac{\mathcal{L}_{2} f(i)}{d_{i}}
\end{aligned}
$$

The second point of view is related to the minimization of equation: $\int|\nabla f(x)|^{p} \mathrm{~d} w$, for which it is well known that the minimum is obtained by having $\Delta_{p} f(x)=0$ for all $x$. On a graph, we can minimize $\sum_{i} \sum_{j \mid j \sim i}\left(f\left(x_{i}\right)-f\left(x_{j}\right)\right)^{p}$ on a graph by equalling to zero the corresponding $p$-Laplacian.

\section{Random walk on a graph and p-Laplacian}

In the continuous case, the p-Laplacian is defined as:

$$
\begin{aligned}
\Delta_{p} f & =\operatorname{div}\left(|\nabla f|^{p-2} \cdot \nabla f\right) \\
& =|\nabla f|^{p-2}\left\{\Delta f+(p-2) \Delta_{\infty} f\right\}
\end{aligned}
$$

where $\Delta$ is the usual $2-$ Laplacian and $\Delta_{\infty}=|\nabla f|^{-2} \frac{1}{2}\left\langle\nabla f, \nabla|\nabla f|^{2}\right\rangle$. Indeed,

$$
\begin{aligned}
\Delta_{p} f & =|\nabla f|^{p-2} \Delta f+(p-2) \sum_{i, j=1}^{n} \frac{|\nabla f|^{p-3}}{|\nabla f|} \frac{\partial f}{\partial x_{i}} \frac{\partial f}{\partial x_{j}} \frac{\partial^{2} f}{\partial x_{i} \partial x_{j}} \\
& =|\nabla f|^{p-2}\left(\Delta f+|\nabla f|^{-2} \frac{1}{2}\left\langle\nabla f, \nabla|\nabla f|^{2}\right\rangle\right) \\
& =|\nabla f|^{p-2}\left\{\Delta f+(p-2) \Delta_{\infty} f\right\}
\end{aligned}
$$

Then, we can define the discretized version on a graph $g$ as $\Delta_{p}^{g} f=\Delta^{g} f+(p-2) \Delta_{\infty}^{g} f$ or put it in a normalized way:

$$
\frac{\Delta^{g} f}{p-1}+\frac{p-2}{p-1} \Delta_{\infty}^{g} f
$$

So, now if we want to minimize $\Delta_{p} f$ over a graph we just need to take discrete versions of $\Delta$ and $\Delta_{\infty}$. The first one is well known and given by:

$$
\Delta_{p}^{g} f(i)=\sum_{k \mid k \sim i} \frac{w_{i k}}{d_{i}}(f(k)-f(i))
$$

The usual random walk on graph is such that if $R_{i j}^{t}$ is the probability of arriving at $j$ in $t$ time steps starting from $i$, for all $i \neq j R_{i j}^{t+1}$ verifies:

$$
\sum_{k \mid k \sim i} \frac{w_{i k}}{d_{i}}\left(R_{k j}^{t}-R_{i j}^{t+1}\right)=0
$$

so that

$$
R_{i j}^{t+1}=\sum_{k \mid k \sim i} \frac{w_{i k}}{d_{i}} R_{k j}^{t}
$$


About, the infinity Laplacian, one of its discrete expressions is:

$$
\Delta_{\infty}^{g} f(u)=\frac{1}{2}\left(\max _{v \sim u} f(v)+\min _{v \sim u} f(v)-2 f(u)\right)
$$

as given in Elmoataz, Desquesnes, and Lézoray (2012), this can be obtained by considering the infinity Laplacian as the second derivative along the steepest direction.

So now, similarly we can aim at minimizing:

$$
\sum_{k \mid \sim i} \frac{w_{i k}}{d_{i}}\left|P_{k j}^{t}-P_{i j}^{t+1}\right|^{p}
$$

keeping a $P^{t+1}$ a probability transition matrix.

Without the need of having a probability transition matrix, we could use directly the discrete $p$-Laplacian and have:

$$
\frac{1}{p-1} \sum_{k \mid k \sim i} \frac{w_{i k}}{d_{i}}\left(Q_{k j}^{t}-Q_{i j}^{t+1}\right)+\frac{p-2}{p-1}\left[\max _{k \mid k \sim i} \frac{1}{2} Q_{k j}^{t}+\min _{k \mid k \sim i} \frac{1}{2} Q_{k j}^{t}-Q_{i j}^{t+1}\right]=0
$$

leading to:

$$
Q_{i j}^{t+1}=\frac{1}{p-1} \sum_{k \mid k \sim i} \frac{w_{i k}}{d_{i}} Q_{k j}^{t}+\frac{p-2}{p-1}\left[\max _{k \mid k \sim i} \frac{1}{2} Q_{k j}^{t}+\min _{k \mid k \sim i} \frac{1}{2} Q_{k j}^{t}\right]
$$

The problem with this expression is that we could have, due to the second term, $\sum_{j} Q_{i j}^{t+1}$ different from 1.

Therefore to define a probability transition matrix $P_{i j}^{t+1}$ for time $t$ we can modify slightly the previous equation by writing:

$$
P_{i j}^{t+1}=\frac{1}{p-1} \sum_{k \mid k \sim i} \frac{w_{i k}}{d_{i}} P_{k j}^{t}+\alpha_{i}^{t} \frac{p-2}{p-1} \max _{k \mid k \sim i} P_{k j}^{t}
$$

where $\alpha_{i}^{t}$ are such that $\sum_{j=1}^{n} P_{i j}^{t+1}=1$.

$\alpha_{i}^{t}$ is given by :

$$
\begin{aligned}
\alpha_{i}^{t} & =\left(1-\sum_{j=1}^{n} \frac{1}{p-1} \sum_{k \mid k \sim i} \frac{w_{i k}}{d_{i}} P_{k j}^{t}\right) /\left(\sum_{j=1}^{n} \frac{p-2}{p-1} \max _{k \mid k \sim i} P_{k j}^{t}\right) \\
& =1 /\left(\sum_{j=1}^{n} \max _{k \mid k \sim i} P_{k j}^{t}\right)
\end{aligned}
$$

Lemma 1. $\forall i, t$ we have $0 \leq \alpha_{i}^{t} \leq 1$.

Proof. Positivity of $\alpha_{i}^{t}$ is obvious by construction. Concerning, the other inequality we can sum $P_{i j}^{t+1}$ to prove it, indeed:

$$
\begin{aligned}
& \sum_{j=1}^{n} P_{i j}^{t+1}=\sum_{j=1}^{n} \frac{1}{p-1} \sum_{k \mid k \sim i} \frac{w_{i k}}{d_{i}} P_{k j}^{t}+\sum_{j=1}^{n} \alpha_{i}^{t} \frac{p-2}{p-1} \max _{k \mid k \sim i} P_{k j}^{t} \text { using equation } 2 \\
& \Rightarrow 1 \geq \frac{1}{p-1}+\alpha_{i}^{t} \frac{p-2}{p-1} \max _{k \mid k \sim i} \sum_{j=1}^{n} P_{k j}^{t} \text { exchanging the max and sum terms } \\
& \Rightarrow 1 \geq \alpha_{i}^{t}
\end{aligned}
$$


Lemma 2. $P^{t} \rightarrow \mathbf{1} v^{\top}$ for some vector $v$.

Proof. $\forall j$, let us consider $i^{*}=\arg \max _{i} P_{i j}^{t}$. Then,

$$
\begin{aligned}
P_{i j}^{t+1} & =\frac{1}{p-1} \sum_{k \mid k \sim i} \frac{w_{i k}}{d_{i}} P_{k j}^{t}+\alpha_{i}^{t} \frac{p-2}{p-1} \max _{k \mid k \sim i} P_{k j}^{t} \\
& \leq \frac{1}{p-1} \sum_{k \mid k \sim i} \frac{w_{i k}}{d_{i}} P_{i^{*} j}^{t}+\alpha_{i}^{t} \frac{p-2}{p-1} P_{i^{*} j}^{t} \\
& \leq P_{i^{*} j}^{t} \text { as } \alpha_{i}^{t} \leq 1
\end{aligned}
$$

This means that for all $j$ the maximum values of $P_{i j}^{t}$ are not increasing. Besides, at one step for a given $j$ either $P_{i_{1} j}^{t}=P_{i_{2} j}^{t}$ for all $i_{1}, i_{2}$ or there exists $i^{*}$ such that for all its neighbours $i$, $P_{i^{*} j}^{t} \geq P_{i j}^{t}$ and there exists $i^{-}$among its neighbours such that $P_{i^{*} j}^{t}>P_{i^{-} j}^{t}$ so that we obtain at next step:

$$
\begin{aligned}
P_{i^{*} j}^{t+1} & =\frac{1}{p-1} \sum_{k \mid k \sim i^{*}} \frac{w_{i^{*} k}}{d_{i^{*}}} P_{k j}^{t}+\alpha_{i^{*}}^{t} \frac{p-2}{p-1} \max _{k \mid k \sim i^{*}} P_{k j}^{t} \\
& <\frac{1}{p-1} \sum_{k \mid k \sim i^{*}} \frac{w_{i^{*} k}}{d_{i^{*}}} P_{i^{*} j}^{t}+\alpha_{i^{*}}^{t} \frac{p-2}{p-1} P_{i^{*} j}^{t} \\
& <P_{i^{*} j}^{t} \text { as } \alpha_{i^{*}}^{t} \leq 1
\end{aligned}
$$

This shows that the maximum values of $P_{i j}^{t}$ are all strictly decreasing for all $j$. Consequently, as they are bounded below by 0 we obtain then convergence.

In general with our setting it is difficult to guess what the stationary distribution is, contrary to what happens with the canonical random walk. However, in next lemma we show that for a $d$-regular graph the stationary distribution is known.

Lemma 3. For a d-regular graph, the stationary distribution is given by $\frac{1}{n} \mathbf{1}$.

Proof. Indeed, if $\forall i, j P_{i j}^{t}=\frac{1}{n}$, we obtain:

$$
\begin{aligned}
P_{i j}^{t+1} & =\frac{1}{p-1} \sum_{k \mid k \sim i} \frac{1}{d} P_{k j}^{t}+\alpha_{i}^{t} \frac{p-2}{p-1} \max _{k \mid k \sim i} P_{k j}^{t} \\
& =\frac{1}{p-1} \sum_{k \mid k \sim i} \frac{1}{d} \frac{1}{n}+\alpha_{i}^{t} \frac{p-2}{p-1} \max _{k \mid k \sim i} \frac{1}{n} \\
& =\frac{1}{p-1} \frac{1}{n}+\alpha_{i}^{t} \frac{p-2}{p-1} \frac{1}{n}
\end{aligned}
$$

Obviously, in that case $\alpha_{i}^{t}=1$ so that: $P_{i j}^{t+1}=\frac{1}{n}$ concluding thus the proof.

Thus, our proposed random walk shares the same stationary distribution with the canonical random walk if the graph is regular.

\section{Application to video games}

If we consider the graph on figure 1 , the transition probabilities $P_{1 i}^{10}$ after 10 steps for state 1 are represented in tables 1 and 2 for $p=2$ and $p=3$ respectively. We observe that the mixing occurs more quickly for $p=3$. That is, for the same number of steps, vertices 11 to 20 are more easily reached with the modified random walk than with the canonical one $(p=2)$. Afterwards, we have compared the convergence speed for the random walks on two differents random graphs. The first type is a set of random barbell graphs where in both cliques of size 
100, edges are randomly deleted with probability 0.5 . The second type is a set of random 3 -regular graphs of size 200 vertices. Convergence is assessed by computing the mean square error among columns of $P^{10}$. Results in table 3 show that in both cases convergence occurs more quickly for $p=3$.

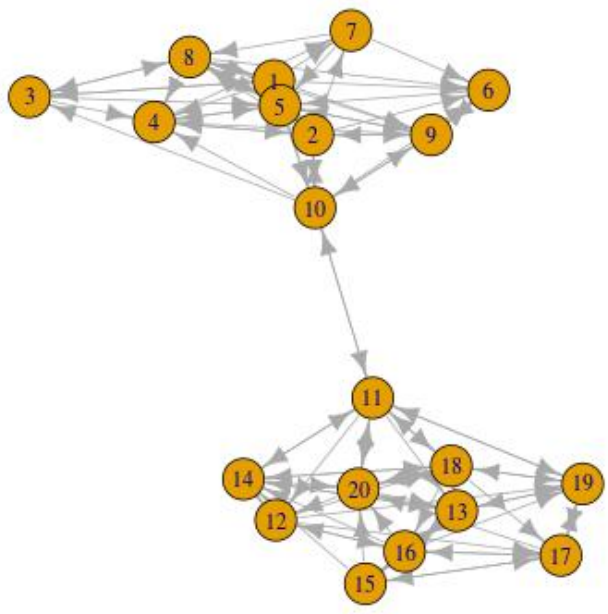

Figure 1: Small graph example drawn using R (R Development Core Team 2008) package igraph (Csardi and Nepusz 2006)

Table 1: Transition probabilities of state 0 after 10 steps for $p=2$.

\begin{tabular}{|c|c|c|c|c|c|c|c|c|c|c|}
\hline State & 1 & 2 & 3 & 4 & 5 & 6 & 7 & 8 & 9 & 10 \\
Proba. & 0.04 & 0.10 & 0.03 & 0.10 & 0.15 & 0.08 & 0.05 & 0.07 & 0.11 & 0.07 \\
\hline State & 11 & 12 & 13 & 14 & 15 & 16 & 17 & 18 & 19 & 20 \\
Proba. & 0.03 & 0.02 & 0.02 & 0.02 & 0.01 & 0.02 & 0.02 & 0.03 & 0.02 & 0.02 \\
\hline
\end{tabular}

Table 2: Transition probabilities of state 0 after 10 steps for $p=3$.

\begin{tabular}{|c|c|c|c|c|c|c|c|c|c|c|}
\hline State & 1 & 2 & 3 & 4 & 5 & 6 & 7 & 8 & 9 & 10 \\
Proba. & 0.04 & 0.06 & 0.03 & 0.06 & 0.09 & 0.06 & 0.05 & 0.06 & 0.08 & 0.05 \\
\hline State & 11 & 12 & 13 & 14 & 15 & 16 & 17 & 18 & 19 & 20 \\
Proba. & 0.04 & 0.04 & 0.05 & 0.04 & 0.04 & 0.04 & 0.04 & 0.05 & 0.05 & 0.04 \\
\hline
\end{tabular}

\section{Conclusion}

We have defined a new random walk, derived from the $p$-Laplacian similary to what is done for the canonical random walk derived from the 2-Laplacian. It is proven to have a stationary distribution which is the same as for the canonical random walk in the case of regular graphs. Experiments demonstrates quicker convergence. Natural perspectives would be to study the convergence speed and also understand what happens between two time steps as what is presented is not a Markov chain. 
Table 3: Experimental comparison of convergence speeds between 2-Laplacian and 3Laplacian random walks after 10 steps.

\begin{tabular}{|c|c|c|c|c|}
\hline & \multicolumn{2}{|c|}{ Random barbell graphs } & \multicolumn{2}{c|}{ 3-Regular graph } \\
& Mean & Sd & Mean & Sd \\
\hline Laplacian & $2.5 * 10^{-5}$ & $4.4 * 10^{-8}$ & $3.1 * 10^{-7}$ & $8.6 * 10^{-8}$ \\
3-Laplacian & $1.5 * 10^{-6}$ & $1.1 * 10^{-7}$ & $1.1 * 10^{-7}$ & $2.6 * 10^{-8}$ \\
\hline
\end{tabular}

\section{Acknowledgements}

Part of this work was done between April and July 2017, when N. Wicker was invited professor at the Kyoto University Bioinformatics Center (Institute for Chemical Research).

\section{References}

Csardi G, Nepusz T (2006). "The Igraph Software Package for Complex Network Research." InterJournal, Complex Systems, p. 1695. URL http://igraph.org.

Elmoataz A, Desquesnes X, Lézoray O (2012). "Non-Local Morphological PDEs and pLaplacian Equation on Graphs with Applications in Image Processing and Machine Learning." IEEE Journal of Selected Topics in Signal Processing, IEEE, 6(7), 764-779.

Grimmet GR, Stirzaker DR (2004). Probability and Random Processes. Oxford Press.

R Development Core Team (2008). R: A Language and Environment for Statistical Computing. R Foundation for Statistical Computing, Vienna, Austria. ISBN 3-900051-07-0, URL http://www.R-project.org.

Żuk A, Pak I (2002). "On Kazhdan Constants and Mixing of Random Walks." International Mathematical Research Notes, 36, 1891-1905.

von Luxburg U, Radl A, Hein M (2014). "Hitting and Commute Times in Large Random Neighborhood Graphs." Journal of Machine Learning Research, 15, 1751-1798.

\section{Affiliation:}

Nicolas Wicker

Département de mathématiques

Université de Lille, Cité Scientifique

59655 Villeneuve d'Ascq, France

E-mail: nicolas.wicker@univ-lille.fr

URL: http://labomath.univ-lille1.fr/ wicker/

\section{Austrian Journal of Statistics}

published by the Austrian Society of Statistics

Volume 48

July 2019 http://www.ajs.or.at/

http://www.osg.or.at/

Submitted: 2018-08-18

Accepted: 2019-01-25 